\title{
First-order transitions and triple point on a random p-spin interaction model
}

\author{
J.M. de Araújo, ${ }^{1}$ F.A. da Costa, ${ }^{2}$ and F.D. Nobre ${ }^{2}$ \\ 1 Departamento de Ciências Naturais \\ Universidade Estadual do Rio Grande do Norte, \\ 59610-210 Mossoró - RN Brazil \\ 2 Departamento de Física Teórica e Experimental \\ Universidade Federal do Rio Grande do Norte \\ Campus Universitário - C.P. 1641 \\ 59072-970 Natal-RN Brazil
}

\begin{abstract}
The effects of competing quadrupolar- and spin-glass orderings are investigated on a spin-1 Ising model with infinite-range random $p$-spin interactions. The model is studied through the replica approach and a phase diagram is obtained in the limit $p \rightarrow \infty$. The phase diagram, obtained within replica-symmetry breaking, exhibits a very unusual feature in magnetic models: three first-order transition lines meeting at a commom triple point, where all phases of the model coexist.
\end{abstract}

Keywords: $\quad$ Spin glasses; First-order transitions;

Triple point.

PACS Numbers: 05.70.-a, 05.70.Fh, 64.60.-i 
The mean-field theory of Ising spin glasses is quite well understood at the present [1,2]. Since the pioneering solution of Parisi [3] for the infinite-range interaction Ising spin glass, the so-called Sherrington-Kirkpatrick (SK) model [4], a wide variety of spin-glass systems has been investigated within the replica approach [1,2]. In particular, this method was used to solve a generalization of the SK model with the inclusion of $p$-spin interactions [5], and it was found that the $p \rightarrow \infty$ limit leads to the solution of the random-energy model, introduced earlier and solved by quite different methods [6]. Since then, many other $p$-spin interaction models have been studied, motivated by the fact that they are tractable within mean-field theory, for arbitrary values of $p$, thus rendering it possible to analyse both $p \rightarrow 2$ and $p \rightarrow \infty$ limits [7]. Another fundamental aspect of such models is their striking connection to real structural glasses [8]. Also, it is usually feasible to study the dynamical properties of those models, making it possible to gain some insights in the important ageing phenomena presented by random systems [8,9].

An important class of random systems, with many physical realizations, is that of orientational glasses [10,11]. These systems are usually described in terms of an assembly of discrete spin variables with quadrupolar random interactions. Recently, a $p$-spin interaction orientational glass model was investigated by the replica method [12], and it was shown that Parisi's replica-symmetry-breaking (RSB) scheme could be applied successfully: indeed, it was found that, in the limit $p \rightarrow \infty$, the low-temperature behavior of such model may be properly described through a single-step RSB approach. This conclusion was shown to be true for another class of models which describe multipolar glasses [13].

Despite all the above-mentioned efforts, much less has been studied on magnetic models where different kinds of disorder are present. This represents a very common situation in physical systems, opening a wide variety of new problems to be investigated. For spin-1 Ising variables, a simple model including pairwise dipolar and quadrupolar random interactions led to interesting behavior, with a competition between quadrupolar- and spin-glass orderings [14].

In the present letter we investigate a spin-1 Ising model including both dipolar and quadrupolar random $p$-spin interactions. We consider an infinite-ranged interacting system, consisting of $N$ spins described through the Hamiltonian, 


$$
\mathcal{H}=-\sum_{1 \leq i_{1}<\cdots<i_{p} \leq N} J_{i_{1} i_{2} \cdots i_{p}} S_{i_{1}} S_{i_{2}} \cdots S_{i_{p}}-\sum_{1 \leq i_{1}<\cdots<i_{p} \leq N} K_{i_{1} i_{2} \cdots i_{p}}\left(S_{i_{1}} S_{i_{2}} \cdots S_{i_{p}}\right)^{2},
$$

where each spin variable can assume the values $0, \pm 1$. Both couplings, $J_{i_{1} \cdots i_{p}}$ and $K_{i_{1} \cdots i_{p}}$ are quenched, independent and identically distributed random Gaussian variables, with zero means and variances $J^{2} p ! /\left(2 N^{p-1}\right)$ and $K^{2} p ! /\left(2 N^{p-1}\right)$, respectively.

It should be mentioned that a spin-1 Ising spin-glass model with $p$-spin interactions, under a single-ion anisotropy field $D$ was already studied by Mottishaw [15]; such a model is identical to the present one only for $D=K=0$. Futhermore, the resulting phase diagrams of these two models share some common features, e.g., they both present three distincts phases. However, whereas Mottishaw's model exhibits one first-order and two continuous critical frontiers, we show that the phase diagram of the present model displays no continuous lines, being characterized by three first-order critical frontiers, which meet at a triple point.

Applying the replica method [1, 2] for the model defined through Eq. (1) and following standard procedures, we get the free-energy density

$$
-\beta f=\lim _{n \rightarrow 0} \frac{1}{n} G_{n}\left(q_{a b}, \lambda_{a b}, Q_{a b}, \gamma_{a b}, R_{a}, \xi_{a}\right),
$$

where

$$
\begin{aligned}
G_{n}\left(q_{a b}, \lambda_{a b}, Q_{a b}, \gamma_{a b}, R_{a}, \xi_{a}\right)= & \frac{\beta^{2}}{4} \sum_{a \neq b}\left(J^{2} q_{a b}^{p}+K^{2} Q_{a b}^{p}\right)+\frac{\beta^{2}\left(J^{2}+K^{2}\right)}{4} \sum_{a} R_{a}^{p} \\
& -\frac{1}{2} \sum_{a \neq b}\left(\lambda_{a b} q_{a b}+\gamma_{a b} Q_{a b}\right)-\sum_{a} \xi_{a} R_{a} \\
& +\ln \operatorname{Tr} \exp \left[\frac{1}{2} \sum_{a \neq b}\left(\lambda_{a b} S^{a} S^{b}+\gamma_{a b}\left(S^{a} S^{b}\right)^{2}\right)+\sum_{a} R_{a}\left(S^{a}\right)^{2}\right],
\end{aligned}
$$

with $\beta=\left(k_{B} T\right)^{-1}$, and $a, b=1 \ldots n$ denoting replica indices. The quantities $\left(\lambda_{a b}, \gamma_{a b}, \xi_{a}\right)$ represent Lagrange multipliers, allowing to fix the set of order parameters $\left(q_{a b}, Q_{a b}, R_{a}\right)$. By demanding $G_{n}$ to be stationary with respect to each of those parameters, we get the equilibrium conditions

$$
\begin{aligned}
& q_{a b}=\left\langle S^{a} S^{b}\right\rangle, \quad Q_{a b}=\left\langle\left(S^{a} S^{b}\right)^{2}\right\rangle, \quad R_{a}=\left\langle\left(S^{a}\right)^{2}\right\rangle, \\
& \lambda_{a b}=\frac{p \beta^{2} J^{2}}{2} q_{a b}^{p-1}, \quad \gamma_{a b}=\frac{p \beta^{2} K^{2}}{2} Q_{a b}^{p-1}, \quad \xi_{a}=\frac{p \beta^{2}\left(J^{2}+K^{2}\right)}{4} R_{a}^{p-1} .
\end{aligned}
$$


Throughout most of this letter, we will be interested in the limit $p \rightarrow \infty$. This is justified by the fact that $p$-interaction models usually exhibit the same qualitative behavior for finite values of $p(p>2)$. Besides that, finite values of $p$ require a substantial amount of numerical work, whereas in the limit $p \rightarrow \infty$ most of the calculations may be carried analytically, based on the fact that the energies of distinct configurations are uncorrelated [5].6].15].

As a preliminary approach to the problem, let us consider the replica-symmetric (RS) solution, i.e., $q_{a b}=q, \quad Q_{a b}=Q, \quad R_{a}=R, \quad \lambda_{a b}=\lambda, \quad \gamma_{a b}=\gamma, \quad \xi_{a}=\xi$, in terms of which the equilibrium equations become

$$
\lambda=\frac{p \beta^{2} J^{2}}{2} q^{p-1}, \quad \gamma=\frac{p \beta^{2} K^{2}}{2} Q^{p-1}, \quad \xi=\frac{p \beta^{2}\left(J^{2}+K^{2}\right)}{4} R^{p-1},
$$

with

$$
q=\left\langle\varphi_{1}^{2}\right\rangle_{x y}, \quad Q=\left\langle\varphi_{2}^{2}\right\rangle_{x y}, \quad R=\left\langle\varphi_{2}\right\rangle_{x y}
$$

In the equations above, $\langle(\ldots)\rangle_{x y}$ stand for $\iint_{-\infty}^{+\infty} \frac{d x d y}{2 \pi} \exp \left(-\frac{x^{2}+y^{2}}{2}\right)(\ldots)$, and

$$
\varphi_{1}=\frac{2 \mathrm{e}^{\delta} \sinh (\sqrt{\lambda} x)}{Z}, \quad \varphi_{2}=\frac{2 \mathrm{e}^{\delta} \cosh (\sqrt{\lambda} x)}{Z},
$$

where

$$
\delta=-\frac{\lambda+\gamma}{2}+\xi+\sqrt{\lambda} y, \quad Z=1+2 \mathrm{e}^{\delta} \cosh (\sqrt{\lambda} x)
$$

One may easily see that the order parameters $Q$ and $R$ never vanish and that Eqs. (5) and (6) always present a trivial solution with $q=\lambda=0$, for arbitrary values of $p$; herein we identify such a solution with a quadrupolar-glass (QG) phase [14.

In the limit $p \rightarrow \infty$ the QG solution represents the only acceptable solution associated with the parameter $q$, since any solution with $q \neq 0$ is unstable, similarly to what happens in the corresponding spin- $\frac{1}{2}$ model [7]; however, there are two simple solutions for the parameters $Q$ and $R$, as we describe below. The first one is given by $\gamma=\xi=0$, and $Q=R^{2}=4 / 9$, in which case the free-energy density becomes

$$
f=-k_{B} T \ln 3
$$


corresponding to an entropy per spin $s=k_{B} \ln 3$; we shall refer to the phase described by such a solution as a quadrupolar-glass 1 (QG1). A second solution can easily be found with $Q=R=1,2 \gamma=\beta^{2} K^{2} p$, and $4 \xi=\beta^{2}\left(J^{2}+K^{2}\right) p$, in such a way as to yield a free-energy density,

$$
f=-\frac{J^{2}}{4 k_{B} T}-k_{B} T \ln 2 .
$$

This solution, which we call quadrupolar-glass 2 (QG2), presents an entropy per spin which becomes negative for $k_{B} T / J<k_{B} T_{c} / J=\frac{1}{2 \sqrt{\ln 2}}=0.6005 \cdots$. For $T>T_{c}$, both solutions are stable, the former one (QG1) presenting a lower free energy at high temperatures. As the temperature is lowered, we find a first-order transition line, where the free energies of those solutions coincide; this line is independent of $K$ and is given by $k_{B} T_{1} / J=\frac{1}{2 \sqrt{\ln (3 / 2)}}=0.7852 \cdots$. It is important to mention that we have also found other solutions, all of them being completely unstable. Therefore, in the limit $p \rightarrow \infty$ the RS solution leads to the phase diagram exhibited in Fig. 1, with two quadrupolar-glass phases, QG1 $(q=0, Q=4 / 9, R=2 / 3)$ and QG2 $(q=0, Q=R=1)$, separated by a first-order transition line.

Since the RS Ansatz leads to a QG2 solution which becomes unstable at low temperatures, one must carry on with a RSB procedure. In analogy with the spin- $\frac{1}{2}$ corresponding problem [0],7] and other $p$-interaction orientational glasses [12,13], one may see that it is sufficient to consider a single-step Parisi RSB scheme for the present problem. This is achieved by grouping the $n$ replicas into $n / m$ blocks of $m$ replicas each. Order parameters with replica indices $a, b$ in the same block take on certain values $\left(q_{a b}=q_{1}, Q_{a b}=Q_{1}, \lambda_{a b}=\lambda_{1}, \gamma_{a b}=\gamma_{1}\right)$, distinguished from those with replica indices in different blocks $\left(q_{a b}=q_{0}, Q_{a b}=Q_{0}, \lambda_{a b}=\lambda_{0}, \gamma_{a b}=\gamma_{0}\right)$, whereas the single-replica-index parameters are considered in the $\mathrm{RS}$ approximation $\left(R_{a}=R, \xi_{a}=\xi\right)$. As usual [3], in the limit $n \rightarrow 0$ the parameter $m$ becomes a continuous variable in the interval $[0,1]$. For an arbitrary value of $p$, the free-energy density becomes

$$
\begin{gathered}
\beta f=-\frac{(\beta J)^{2}}{4}\left[(m-1) q_{1}^{p}-m q_{0}^{p}\right]-\frac{(\beta K)^{2}}{4}\left[(m-1) Q_{1}^{p}-m Q_{0}^{p}\right]-\frac{\beta^{2}\left(J^{2}+K^{2}\right)}{4} R^{p} \\
+\frac{1}{2}\left[(m-1)\left(\lambda_{1} q_{1}+\gamma_{1} Q_{1}\right)-m\left(\lambda_{0} q_{0}+\gamma_{0} Q_{0}\right)\right]+\xi R \\
+\frac{1}{m} \int_{-\infty}^{\infty} \int_{-\infty}^{\infty} \frac{d x_{0} d x_{1}}{2 \pi} \exp \left(-\frac{x_{0}^{2}+x_{1}^{2}}{2}\right) \ln Z\left(x_{0}, x_{1}\right),
\end{gathered}
$$


where

$$
Z\left(x_{0}, x_{1}\right)=\frac{1}{m} \int_{-\infty}^{\infty} \int_{-\infty}^{\infty} \frac{d y_{0} d y_{1}}{2 \pi} \exp \left(-\frac{y_{0}^{2}+y_{1}^{2}}{2}\right)\left[\operatorname{Tr}\left(\mathrm{e}^{\mathrm{B}}\right)\right]^{m}
$$

and

$$
B=\left(\sqrt{\lambda_{0}} x_{0}+\sqrt{\lambda_{1}-\lambda_{0}} y_{0}\right) S+\left(\sqrt{\gamma_{0}} x_{1}+\sqrt{\gamma_{1}-\gamma_{0}} y_{1}+\xi-\frac{\lambda_{1}}{2}-\frac{\gamma_{1}}{2}\right) S^{2}
$$

From the above free-energy density functional we can obtain several solutions, including those already described within the RS approximation. From now on, we will restrict ourselves to the limit $p \rightarrow \infty$. Our analysis indicates that there is only one new solution within a one-step RSB that is physically acceptable, i.e., $q_{0}, Q_{0}<1, q_{1}=Q_{1}=R=1$. This solution presents a free-energy density independent of $T$, given by

$$
f=-\sqrt{\left(J^{2}+K^{2}\right) \ln 2},
$$

with a zero entropy. It corresponds to the low-temperature phase, where all spin variables are frozen completely at random, each of them in one of the states $S_{i}= \pm 1$, like in a spin- $\frac{1}{2}$ Ising spin glass. Such a solution will be associated with the quadrupolar-spin-glass (QSG) phase.

Since we have obtained three phases (QG1, QG2 and QSG) and their respective free energies, we can draw the phase diagram of the model within the RSB approach (see Fig. 2). We adopt the standard thermodynamic criteria, i.e., whenever two or more solutions are stable, the correct phase is defined as the one with the lowest free energy. Besides the first-order transition line separating phases QG1 and QG2 (which remains a line independent of $K$ at $\left.k_{B} T_{1} / J=0.7852 \cdots\right)$, we find two new first-order transition lines: one represents the coexistence of phases QG2 and QSG and is given by

$$
k_{B} T_{2}=\frac{\sqrt{J^{2}+K^{2}}+K}{2 \sqrt{\ln 2}},
$$

whereas the other one corresponds to a coexistence of phases QG1 and QSG,

$$
k_{B} T_{3}=\frac{\sqrt{\left(J^{2}+K^{2}\right) \ln 2}}{\ln 3} .
$$

The three phases coexist at a triple point, whose coordinates are given by 


$$
k_{B} T_{t} / J=\frac{1}{2 \sqrt{\ln (3 / 2)}}=0.7852 \cdots, \quad K_{t} / J=\frac{2 \ln 2-\ln 3}{2 \sqrt{\ln 2 \ln (3 / 2)}}=0.2713 \cdots
$$

where all three lines merge together, in accordance with the famous Gibbs phase rule [16].

In conclusion, we have solved a disordered spin-1 Ising model with $p$-spin interactions through the replica method. Considering the limit $p \rightarrow \infty$, we have verified that the Parisi Ansatz is suitable to determine correctly a phase diagram with genuine first-order transitions, each of them accompanied by a latent heat and exhibiting discontinuities on the respective order parameters. Each phase is described by the solution which is a global minimum of the free energy. A very uncommon feature in magnetic models has been detected, i.e., a triple point where all three phases coexist. An important aspect to be explored is the dynamics of this model, mainly along the first-order transition lines; this issue is currently been investigated.

\section{Acknowledgments}

One of us (FDN) thanks CNPq and Pronex/MCT (Brazil) for partial financial support. 


\section{REFERENCES}

[1] Binder K. and Young A.P., Rev. Mod. Phys., 58 (1986) 801.

[2] Fischer K.H. and Hertz J.A., Spin Glasses (Cambridge University Press, Cambridge) 1991.

[3] Parisi G. , Phys. Rev. Lett., 43 (1979) 1754; J. Phys. A.: Math. Gen., 13 (1980) L115, 1101, 1887.

[4] Sherrington D. and Kirkpatrick S., Phys. Rev. Lett., 35 (1975) 1792.

[5] Gross D.J. and Mézard M., Nucl. Phys. B, 240 (1984) 431.

[6] Derrida B. , Phys. Rev. Lett., 45 (1980) 79; Phys. Rev., B 24 (1981) 2613.

[7] Gardner E., Nucl. Phys. B, 257 (1985) 747.

[8] Kirkpatrick T.R. and Thirumalai D., Phys. Rev. Lett., 58 (1987) 2091; Phys. Rev. $B, 36$ (1987) 5388.

[9] Bouchaud J.-P., Cugliandolo L.F., Kurchan J. and Mézard M., in Spin Glasses and Random Fields, edited by A.P. Young (World Scientific, Singapore) 1998, pp. 161-223.

[10] Höchli U.T., Knorr K. and Loidl A., Adv. Phys., 39 (1990) 409.

[11] Binder K. and Reger J.D., Adv. Phys. 41, (1992) 547; Binder K., in Spin Glasses and Random Fields, edited by A.P. Young (World Scientific, Singapore) 1998, pp. 99-118.

[12] Walasek K., J. Phys. A: Math. Gen., 28 (1995) L497.

[13] Kopeć T.K., J. Phys. A: Math. Gen., 29 (1996) L49.

[14] de Araújo J.M. , da Costa F.A. and Nobre F.D., "Competition Between Quadrupolarand Spin-Glass Orderings in Spin-1 Systems", Preprint, 1999.

[15] Mottishaw P., Europhys. Lett., 1 (1986) 409.

[16] Callen H.B., Thermodynamics and an Introduction to Thermostatistics, Second Edition (John Wiley and Sons, Inc., New York) 1985. 


\section{FIGURES}

FIG. 1. The phase diagram within the RS approximation. The quadrupolar-glass phases, QG1 and QG2 (defined in the text), are separated by a first-order transition line. Throughout the gray region the solution QG2 becomes unstable, presenting a negative entropy.

FIG. 2. The phase diagram within Parisi's RSB procedure. All phase boundaries are first-order transition lines. At the triple point (black dot) the three phases coexist. 


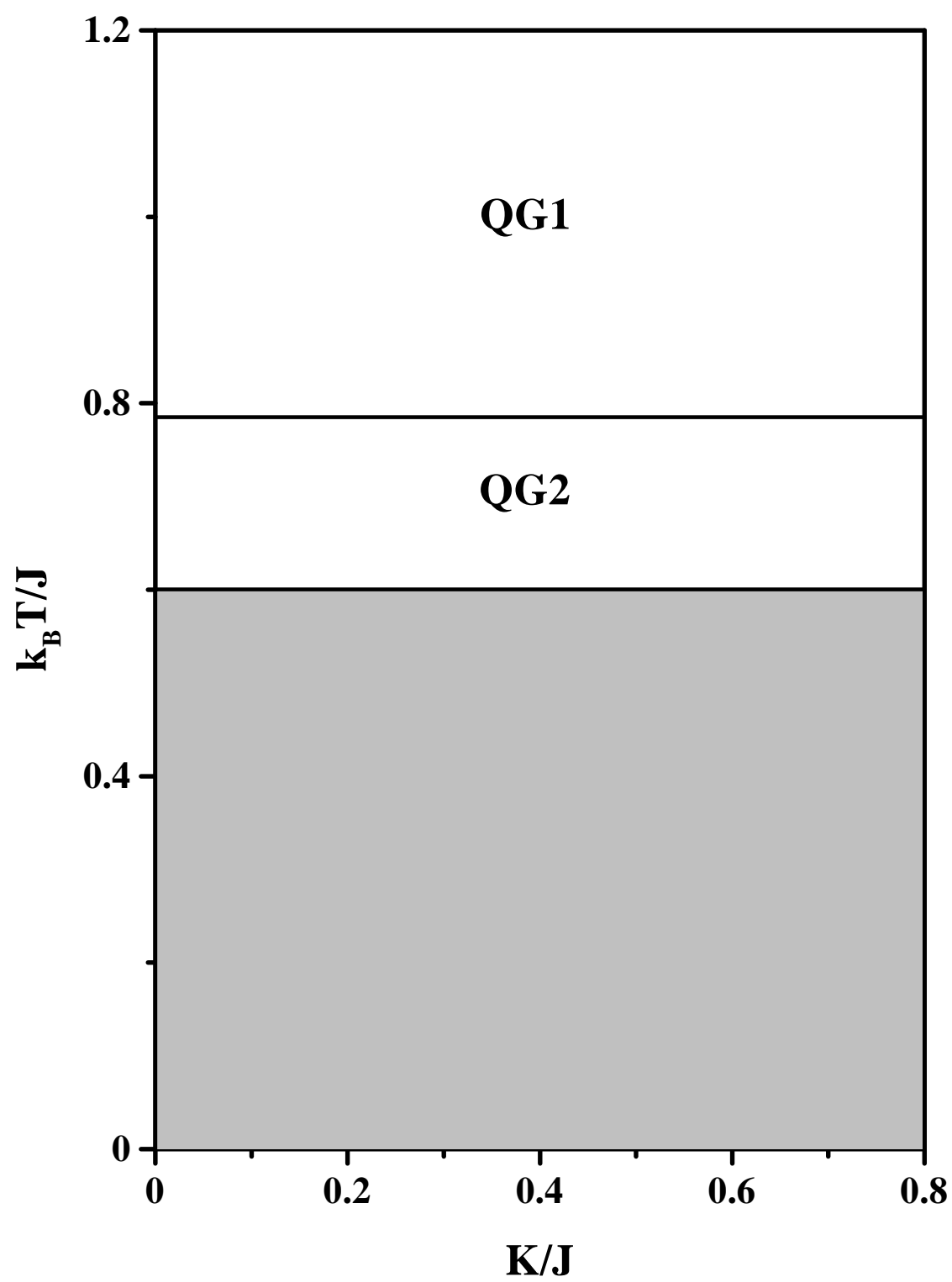

Fig. 1 - de Araújo et al. 


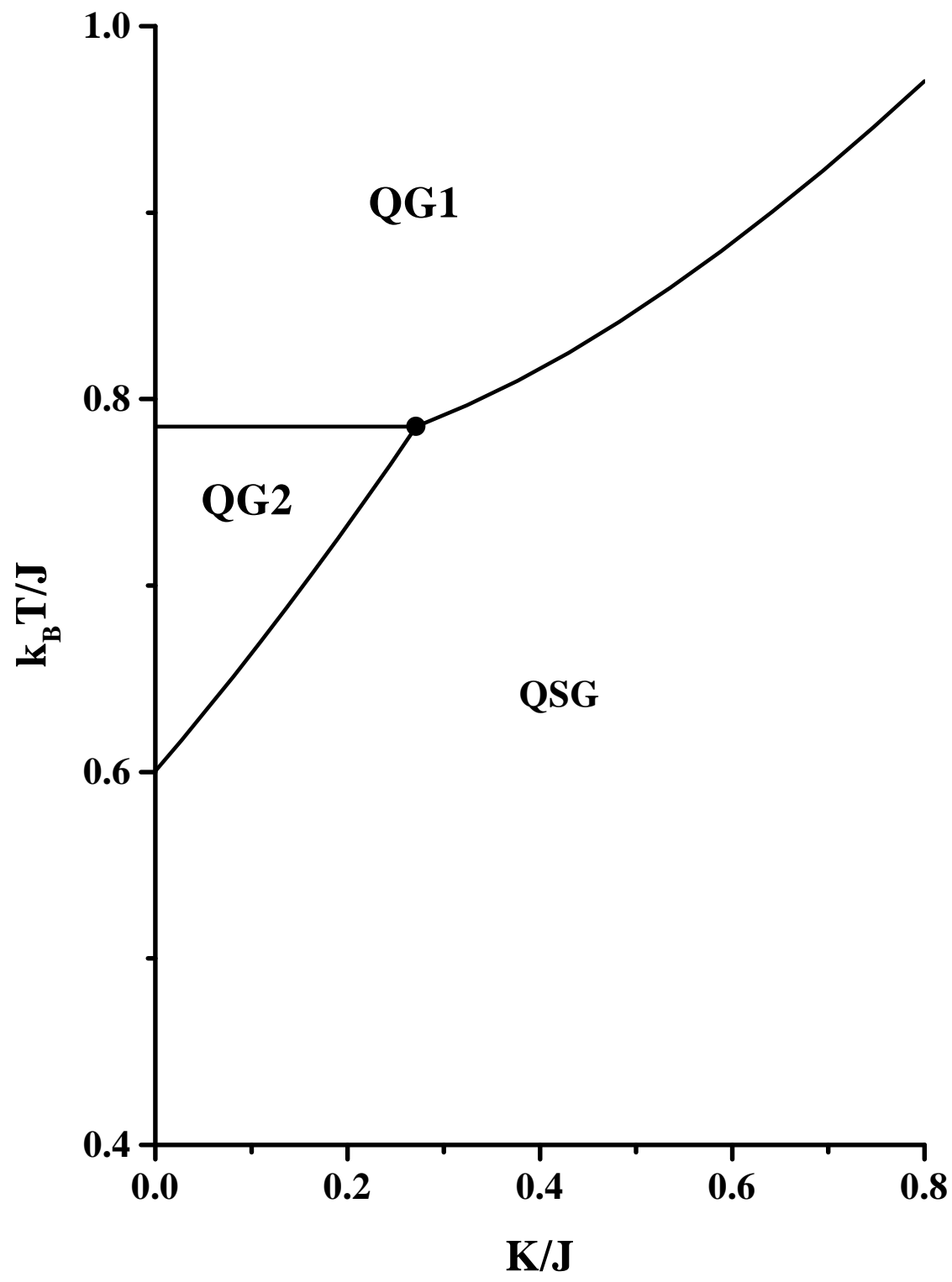

Fig. 2 - de Araújo et al. 